\title{
Low Model Analysis and Synchronous Simulation of the Wave Mechanics
}

\author{
Wenyuan Duan, Heyuan Wang, and Meng Kan \\ College of Science, Liaoning University of Technology, Jinzhou 121001, China \\ Correspondence should be addressed to Wenyuan Duan; 1499206003@qq.com
}

Received 11 May 2016; Accepted 13 July 2016

Academic Editor: Bernard Bonello

Copyright (C) 2016 Wenyuan Duan et al. This is an open access article distributed under the Creative Commons Attribution License, which permits unrestricted use, distribution, and reproduction in any medium, provided the original work is properly cited.

The dynamic behavior of a chaotic system in the internal wave dynamics and the problem of the tracing and synchronization are investigated, and the numerical simulation is carried out in this paper. The globally exponentially attractive set and positive invariant set of the chaotic system are studied via constructing the positive definite and radial unbounded Lyapunov function. There are no equilibrium positions, periodic solutions, quasi-period motions, wandering recovering motions, and other chaotic attractors of the system out of the globally exponentially attractive set. Strange attractors can only locate in the globally exponentially attractive set. A feedback controller is designed for the chaotic system to realize the control of the unstable point. The second method of Lyapunov is used to discuss theoretically the rationality of the design of the controller. The driving-response synchronization method is used to realize the globally exponential synchronization. The numerical simulation is carried out by MATLAB software, and the simulation results show that the method is effective.

\section{Introduction}

Since 1960s, in the meteorological numerical research conducted by American meteorologist Lorenz, the threedimensional truncation is carried out for the RayleighBernard heat convection problem of the infinite dimensional dynamical system to get the famous Lorenz system and the chaos phenomenon is found accidently [1]. A lot of researches on chaos are conducted by many scholars since the discovery of chaos [1-10]. Lorenz system reveals the essential phenomenon of the nonlinear complexity as the first chaotic system $[8,9]$. The chaotic attractor was formally proposed by Ruelle and Takens in 1971 and the nonperiodic flow appearing in the dissipative system was called the strange attractor by them [1]. The capture area of the strange attractor and the global stability of the chaotic system are hot spots of common concern to the people. The low mode analysis is usually used to explain and predict the chaotic phenomenon of infinite dimensional dynamical system [10]. The inertial manifold and approximate inertial manifold theories are the theoretical foundation and basis of the low mode analysis (they are considered to be a low dimensional smooth manifold with exponent attracting all tracks containing the global attractor).
The complex dynamic behavior of the infinite dimensional dynamical system usually originated from a simple origin and can be distinguished by the simple equation. The three functions of the internal gravity wave in the atmosphere are the following [11]: transmission and storage of energy and momentum, starting and organization of the convective activity, and occurrence and modulation of the turbulence. The thermal convection and turbulence caused by the internal wave are studied in essence in Lorenz's paper [10]. The chaos caused by the match of the driving factor with the dissipation factor of the turbulence appears in the internal gravity wave dynamics. The dissipation factor is too weak causing the absence of chaos when the Prandtl number $\sigma$ is equal to 1 . The concept of chaos can help explain the occurrence of atmosphere turbulence. During the day, unstable temperature stratification is the driving factor of turbulence, and the molecular viscosity and certain velocity shear are dissipation factor. At night, effective surface long wave radiation is the driving factor of turbulence. The chaos can still appear under the condition that the real atmosphere Prandtl number is equal to 0.7 after introducing the velocity shear damping. The smaller the Richardson number is, the more likely the chaos appears at night [11]. Chaos is too 
sensitive to the initial value and the unpredictability of the trend of the long time of chaos exists, so the chaos system is considered to be uncontrollable and two chaos systems are considered to be synchronous impossibly by people for a long time. The OGY method was proposed since 1990 and the chaos synchronization [12-15] was used by the United States Navy laboratories to get the secure communication firstly in 1990 that changed people's original idea. The control and synchronization of chaos has become a hot spot in the field of nonlinear science in recent years.

A new chaos system is found by Liu through numerical calculation in the study of the internal wave dynamics [11]. But he did not have a comprehensive analysis of the appearance and disappearance of chaotic attractor. Based on the research of Liu, we have carried out a comprehensive and detailed discussion and numerical simulation in this system; the corresponding attractors of the system are given; at the same time, we obtain the bifurcation diagram, power spectrum, Poincare section, and return mapping of the system. The general characteristics of the behavior of the chaotic system are detected via simulation, and we discuss the global stability of the system. The globally exponentially attractive set and positive invariant set of the system are given by constructing the positive and radial unbounded Lyapunov function. According to the concept of globally exponential tracking, for any periodic solution or equilibrium, we design some simple feedback controllers to control all the trajectories of the chaotic systems tracking the special periodic solution globally. The adaptive control method is used to realize the generalized index synchronization and the effectiveness of the method is validated by the numerical simulation.

\section{Brief Introduction of the Behavior of the Dynamics and Three-Mode System of the Internal Wave Dynamics}

Three-dimensional nonlinear system is achieved by the truncated spectral method in [11] as follows:

$$
\begin{aligned}
& \dot{x}=-(\sigma+s) x+\sigma y, \\
& \dot{y}=r x-y-x z, \\
& \dot{z}=x y-b z,
\end{aligned}
$$

where $\sigma, s$, and $b$ are constants, $r$ is the variational parameter, and $x, y, z$ are the spectral expansion coefficient.

The linear stability analysis of system (1) is carried out by [11] and the main conclusions are as follows:

(1) When $0<r<(\sigma+s) / \sigma$, the equilibrium state $O$ is stable.

(2) When $(\sigma+s) / \sigma<r<r_{0}=380.6$, the equilibrium state $O$ is unstable and the equilibrium states $C_{1}$ and $C_{2}$ are stable, where $\lambda_{1}<0$, the real part of the equation $\lambda_{2}=$ $\lambda_{3}$ is negative, and the pitch fork bifurcation occurs when $r=(\sigma+s) / \sigma$.

(3) When $r>r_{0}$, the equilibrium states $C_{1}$ and $C_{2}$ lose the stability, where $\lambda_{1}<0$. But the real part of the equation $\lambda_{2}=\lambda_{3}$ is positive. The subcritical Hopf bifurcation occurs when $r=r_{0}$.

For system (1), the change rate of the volume in phase space $(x, y, z)$ is

$$
\frac{1}{V} \frac{d V}{d t}=\frac{\partial \dot{x}}{\partial x}+\frac{\partial \dot{y}}{\partial y}+\frac{\partial \dot{z}}{\partial z}=-(\sigma+s+1+b)<0 .
$$

From formula (2), system (1) is the dissipative system of volume shrinkage on the whole, so movement will eventually tend to a certain attractor.

On the basis, in [11], the parameters can be selected as $\sigma=0.7, s=10, b=8 / 3, r=500$, and the initial values can be selected as $x=10, y=100, z=400$, step size $h=0.1$. The simulation calculation and analysis on the internal wave dynamics triple modular system (1) are carried out and the projection of a part of the trajectory in the phase space in the two-dimensional plane is simulated. The space orbit of the three-dimensional space is changed into the one-dimensional map by the Poincare section method. The chaotic phenomenon exists in system (1) when $r>r_{0}$ that is illustrated by analysis and calculation.

\section{Numerical Simulation of the Dynamic Behavior of the System}

More detailed numerical simulation and theoretical analysis of the dynamic behavior of system (1) are carried out in this section. The parameters can be selected as $\sigma=0.7, s=$ $10, b=8 / 3$; with the increase of the $r$, we obtain the attractor diagram, bifurcation diagram of system (1) by simulation, and we obtain the Poincare section, power spectrum, and return mapping of system (1) by simulation, further validating the chaotic behavior of the system. The conclusions combining the simulation results with theoretical analysis are as follows:

(1) When $0<r<r_{1}=15.286 \ldots$, there is only one fixed point $O$, and it is stable.

(2) When $r_{1}<r<r_{e}=237.9 \ldots$, point $O$ becomes the unstable saddle-node point with one unstable direction and two stable directions. The eigenvalues of $C_{1}$ and $C_{2}$ all are negative real roots, so $C_{1}$ and $C_{2}$ are the asymptotically stable nodes.

(3) When $r_{e}<r<r_{0}=380.6 \ldots$, the equation has a negative real root and conjugate complex roots with negative real part that represents the point in one direction of $C_{1}$ and $C_{2}$ being asymptotically stable, while there is stable focus in the plane, which is perpendicular to this direction. Numerical calculation shows that they are global attractors, as shown in Figure 1.

(4) When $r>r_{0}$, there is still a negative real root and others are conjugate complex roots with the positive real part that represents the point in the direction of $C_{1}$ and $C_{2}$ being stable and the focus in the plane vertical to the stable direction is unstable focus. Then Hopf bifurcation [16-18] appears when 


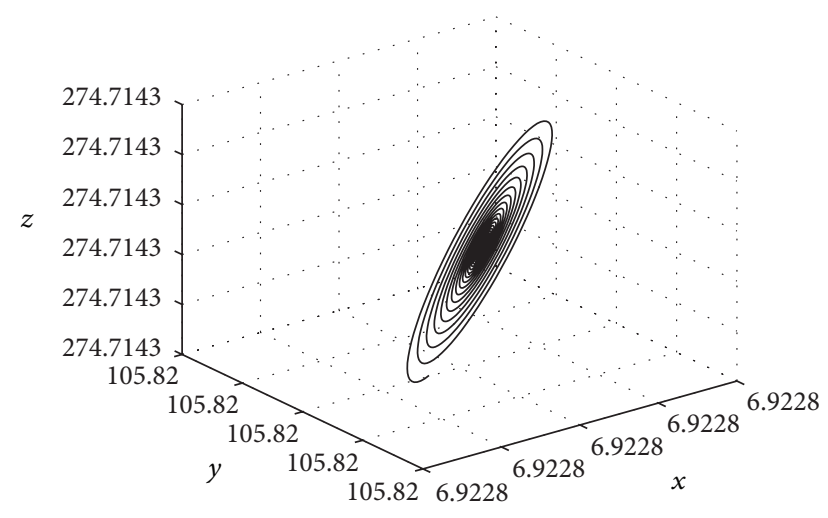

FIGURE 1: $r_{e}<r<r_{0}=380.6 \ldots$

$r=r_{0}$ and strange attractors generated to cause chaos. Eventually, the process of chaos is shown in Figures 2, 3,4 , and 5 .

(5) When $2169.9 \ldots<r<2260.7 \ldots$. Figures 6 and 7 show that the attractor gradually starts to shrink into the limit cycle which is an inverted bifurcation process, and the numerical results show that the bifurcation point satisfies the Feigenbaum constant.

(6) When 2260.7 . $<r<2743.1 \ldots$, chaos appears firstly and then gradually disappears to shrink into the torus which is still an inverted bifurcation process; this process is shown in Figures 8, 9, and 10, and the bifurcation point satisfies the Feigenbaum constant.

(7) The bifurcation diagram of system (1) can be seen in Figure 11. From the simulation results of the image, the whole process from the starting to the end of the chaotic phenomenon can be observed. The Poincare section, power spectrum, and return mapping in Figures 12, 13, and 14 when $r=800$ all show the chaotic character of the system.

\section{The Globally Exponentially Attractive Set and Positive Invariant Set}

In the following we discuss the global stability of the chaotic system (1) in this section to get the estimation of globally exponentially attractive set and the positive invariant set of system (1).

First of all, we give some related definitions [19]. Denote by $X=(x, y, z)$ the state vector of system (1), and $Q \subset R^{3}$ as a compact set (bounded and closed set). Let $t_{0} \geq 0$ be the initial time. By $X\left(t, t_{0}, X_{0}\right)$ we denote the solution of system (1) satisfying the initial value $X\left(t_{0}, t_{0}, X_{0}\right)=X_{0}$. If there is no confusion, we denote this solution by $X(t)$. Define the distance between the solution vector $X\left(t, t_{0}, X_{0}\right)$ and the set $Q$ as $\rho\left(X\left(t, t_{0}, X_{0}\right), Q\right)=\inf _{\widehat{X} \in Q}\left\|X\left(t, t_{0}, X_{0}\right)-\widehat{X}\right\|$. Define $Q_{\varepsilon}$ as any set that includes the set $Q$; that is, $Q \subset Q_{\varepsilon}$.

Definition 1. If there exists a compact set $Q$ in the space $R^{3}$ such that $\rho(X(t), Q) \rightarrow 0$ as $t \rightarrow \infty, \forall X_{0} \in R^{3} / Q$, then one calls the set $Q$ a globally attractive set of system (1). In

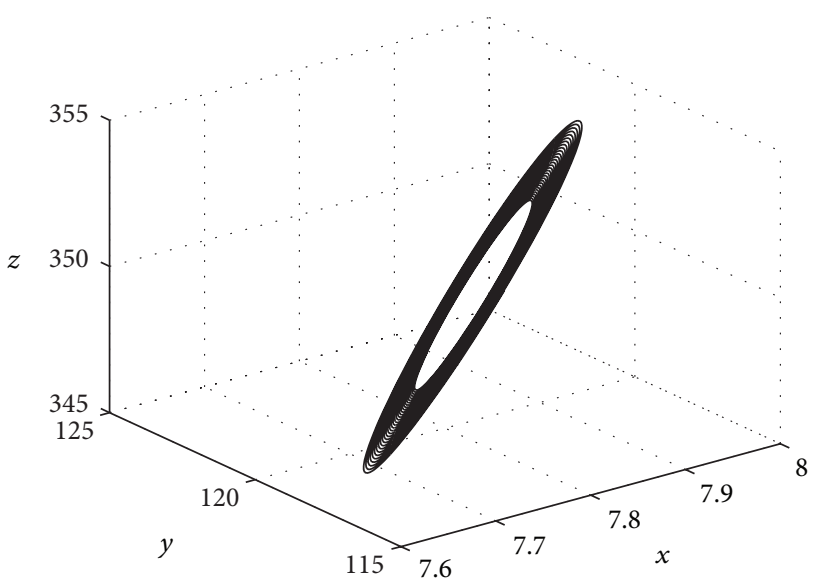

FIGURE 2: $r>r_{0}$.

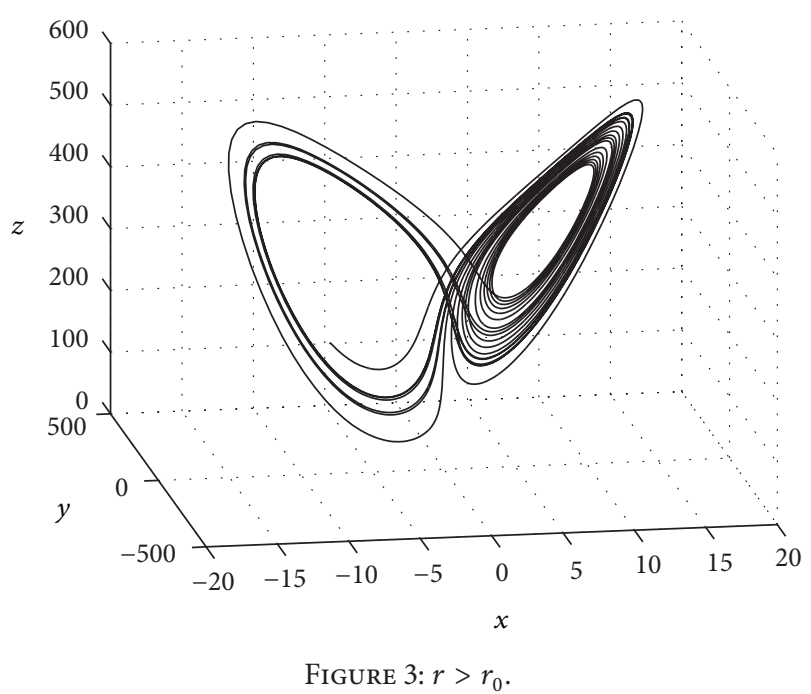

other words, there exists $T>t_{0}$, such that $X\left(t, t_{0}, X_{0}\right) \subseteq Q_{\varepsilon}$ for $t \geq T+t_{0}$. A system with the globally attractive set is called a globally asymptotically Lagrangian stable system or a dissipative system in ultimately bounded sense. If, $\forall X_{0} \in$ $Q, X\left(t, t_{0}, X_{0}\right) \subseteq Q$ holds for all $t \geq t_{0}$, the set $Q$ is called a positively invariant set of system (1).

From the definition, we conclude that if $Q$ is a globally attractive set then all $Q_{\varepsilon}$ are the globally attractive sets too.

Theorem 2. Let $\sigma>0, s \geq 10, b \geq 1, r \geq(\sigma+s) / \sigma$. For any constant $m \geq 0$, ellipsoidal

$$
\Omega_{m}: m x^{2}+y^{2}+(z-m \sigma-r)^{2} \leq \frac{b^{2}(m \sigma+r)^{2}}{2 b-1}
$$

is a globally attractive set and positively invariant set of system (1).

Proof. It is easy to show that if $0<r<(\sigma+s) / \sigma$ the unique equilibrium $(0,0,0)^{T}$ of system (1) is globally exponentially stable. If $r=(\sigma+s) / \sigma$, the equilibrium $(0,0,0)^{T}$ is stable but not asymptotically stable. Now we construct a family of 


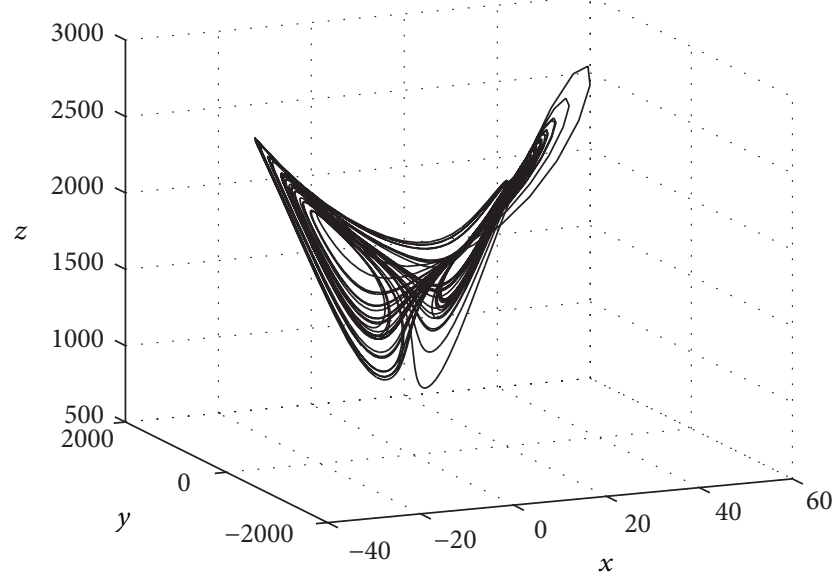

FIGURE 4: $r>r_{0}$.

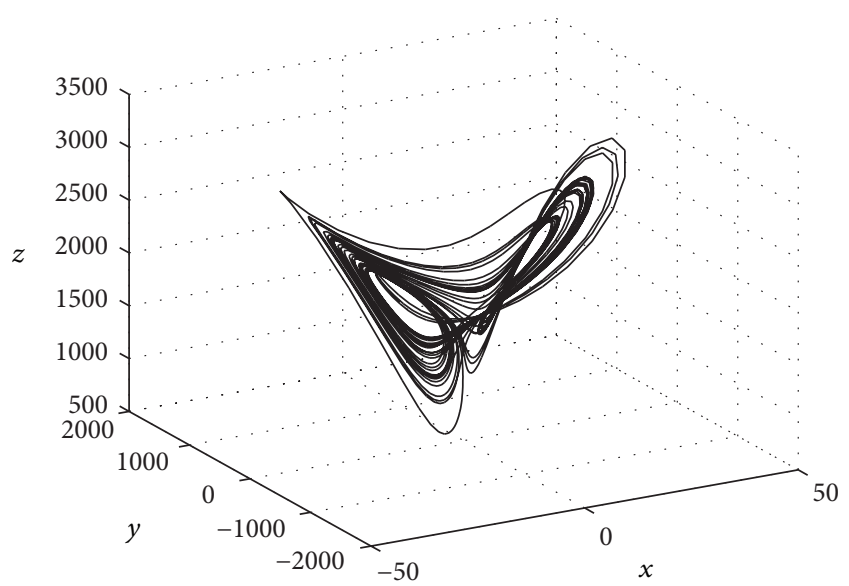

FiguRE 5: $r>r_{0}$.

generalized radically infinite and positive definite Lyapunov functions as

$$
V_{m}(X)=m x^{2}+y^{2}+(z-m \sigma-r)^{2},
$$

when $m \geq 0$ is arbitrary constant. Since $V_{m}(X)=0$ holds for $x=y=0, z=m \sigma+r, V_{m}(X)$ is not positive with respect to the state vector. The point $(0,0, m \sigma+r)^{T}$ is not the equilibrium of system (1). Therefore, the family of Lyapunov functions cannot be employed to study stability of any equilibrium of system (1). So, it is called the family of generalized positive definite Lyapunov functions.

Computing the derivative of $V$ along the positive halftrajectory of system (1), we have

$$
\begin{aligned}
\dot{V}= & 2 m x \cdot \dot{x}+2 y \cdot \dot{y}+2(z-m \sigma-r) \dot{z} \\
= & 2 m x \cdot(-(\sigma+s) x+\sigma y)+2 y \cdot(r x-y-x z) \\
& +2(z-m \sigma-r)(x y-b z) \\
& =-V+\left(-2 m\left(\sigma+s-\frac{1}{2}\right)\right) x^{2}-y^{2}-(2 b-1) z^{2}
\end{aligned}
$$

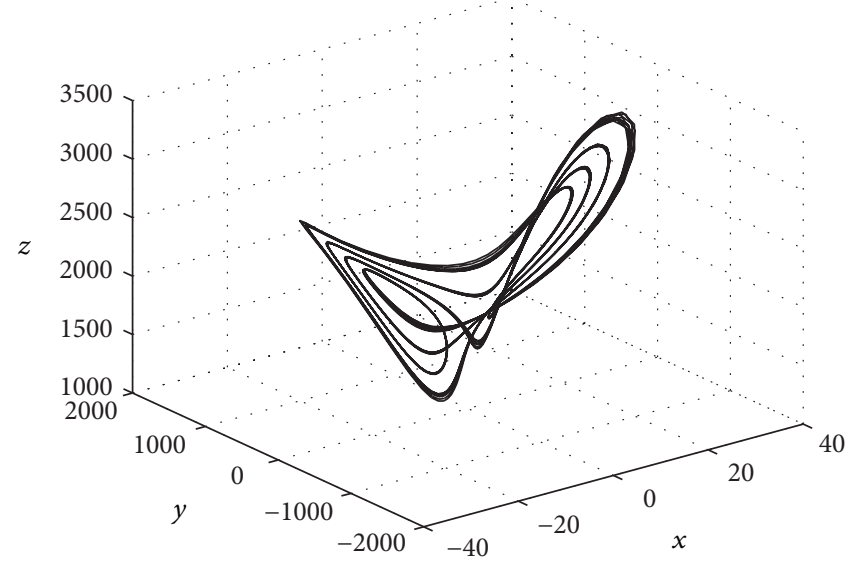

FigURE $6: 2169.9 \ldots<r<2260.7 \ldots \ldots$

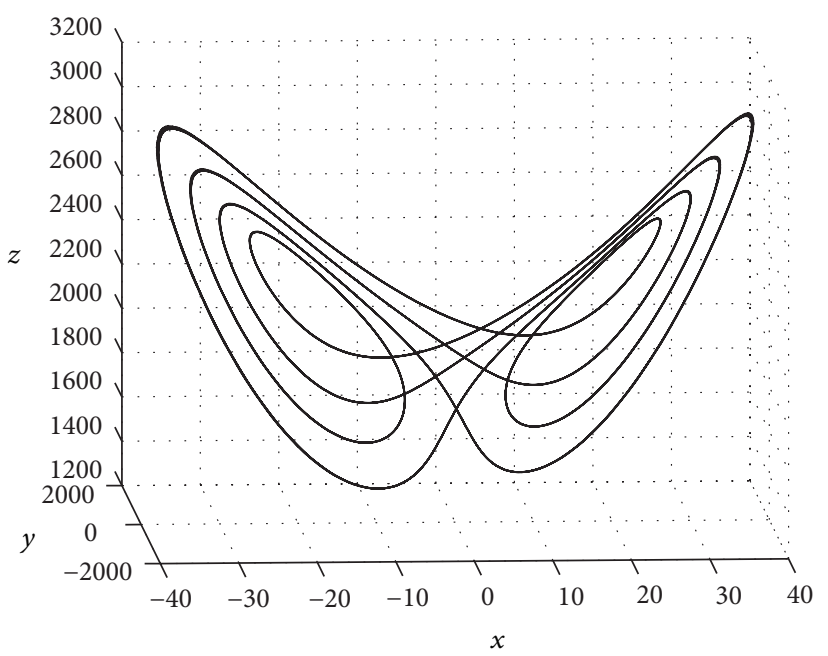

FIGURE 7: $2169.9 \ldots<r<2260.7 \ldots$

$$
\begin{aligned}
& +2(m \sigma+r)(b-1) z+(m \sigma+r)^{2} \\
= & -V+F(X),
\end{aligned}
$$

where $X=(x, y, z)$, and define the function

$$
\begin{aligned}
F(X)= & -2 m\left(\sigma+s-\frac{1}{2}\right) x^{2}-y^{2}-(2 b-1) z^{2} \\
& +2(m \sigma+r)(b-1) z+(m \sigma+r)^{2}
\end{aligned}
$$

computing the extreme value of Lagrange of function $F(X)$ on $(x, y, z)$, because $F(X)$ is the quadratic function and the local maxima are the global maximum, and letting

$$
\begin{aligned}
& \frac{\partial F}{\partial x}=-4 m\left(\sigma+s-\frac{1}{2}\right) x=0, \\
& \frac{\partial F}{\partial y}=-2 y=0, \\
& \frac{\partial F}{\partial z}=-2(2 b-1) z+2(m \sigma+r)(b-1)=0,
\end{aligned}
$$

we have $x=0, y=0, z=(m \sigma+r)(b-1) /(2 b-1)$. 


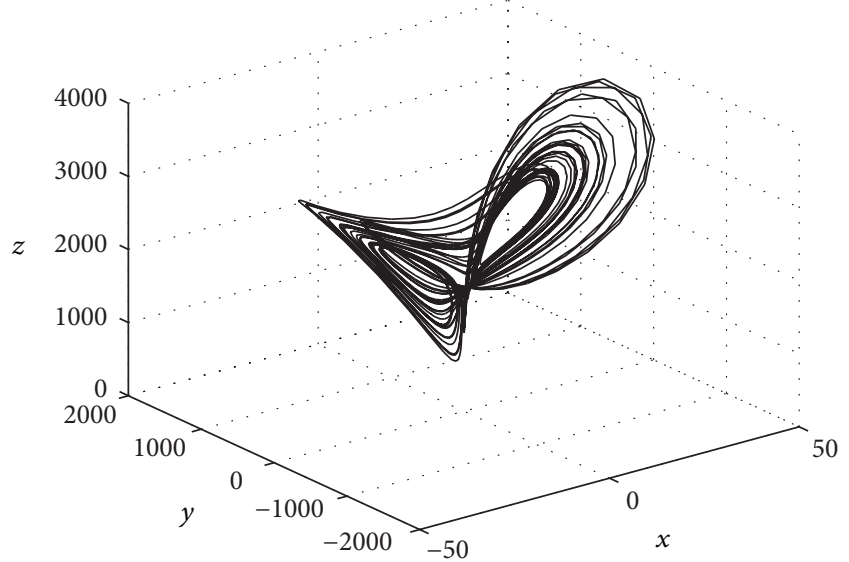

Figure 8: $2260.7 \ldots<r<2743.1 \ldots$

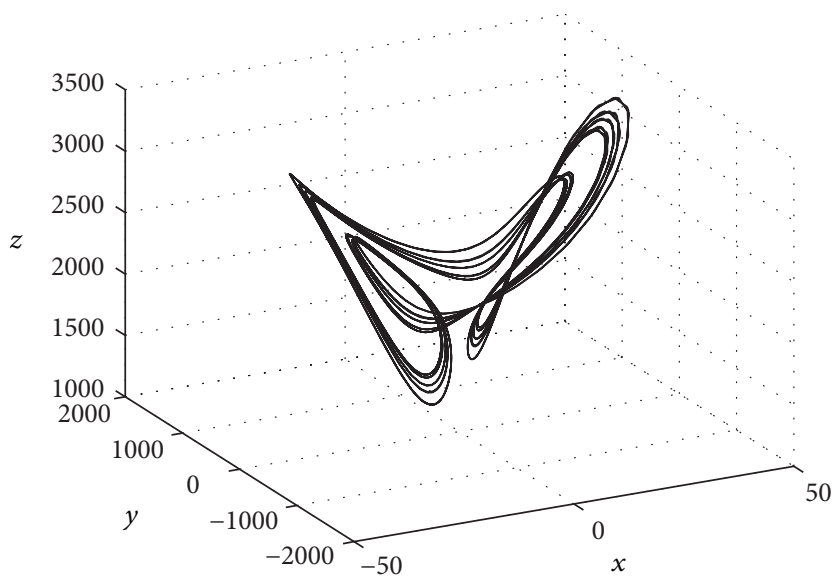

Figure 9: $2260.7 \ldots<r<2743.1 \ldots$.

Then computing the two-order derivative of $F(X)$, we obtain

$$
\begin{aligned}
& \frac{\partial^{2} F}{\partial x^{2}}=-4 m\left(\sigma+s-\frac{1}{2}\right)<0, \quad\left(\text { For } \sigma+s>\frac{1}{2}\right) \\
& \frac{\partial^{2} F}{\partial y^{2}}=-2<0, \\
& \frac{\partial^{2} F}{\partial z^{2}}=-2(2 b-1)<0, \quad\left(\text { For } b>\frac{1}{2}\right) \\
& \frac{\partial^{2} F}{\partial x \partial y}=\frac{\partial^{2} F}{\partial y \partial z}=\frac{\partial^{2} F}{\partial x \partial z}=0,
\end{aligned}
$$

so $\sup _{X \in R^{3}} F(X)=\left.F(x, y, z)\right|_{(x=0, y=0, z=(m \sigma+r)(b-1) /(2 b-1))}=$ $b^{2}(m \sigma+r)^{2} /(2 b-1)$.

When $V>b^{2}(m \sigma+r)^{2} /(2 b-1), \dot{V}<0$, so we have

$$
\lim _{t \rightarrow+\infty} \rho\left(X\left(t, t_{0}, X_{0}\right), \Omega_{m}\right)=0
$$

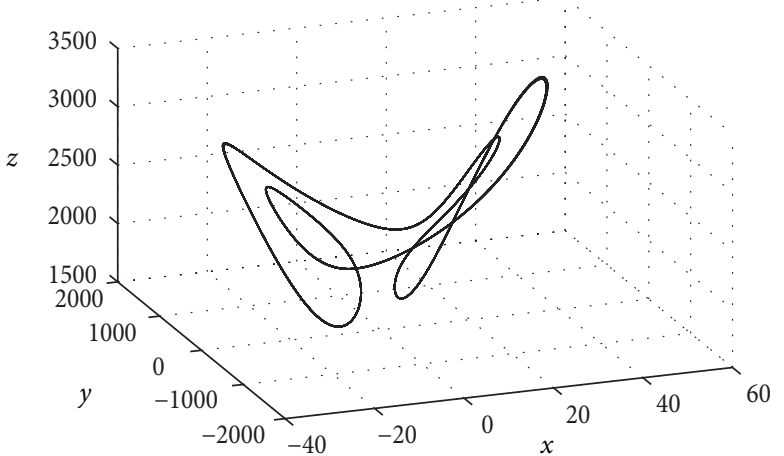

Figure 10: $2260.7 \ldots<r<2743.1 \ldots$.

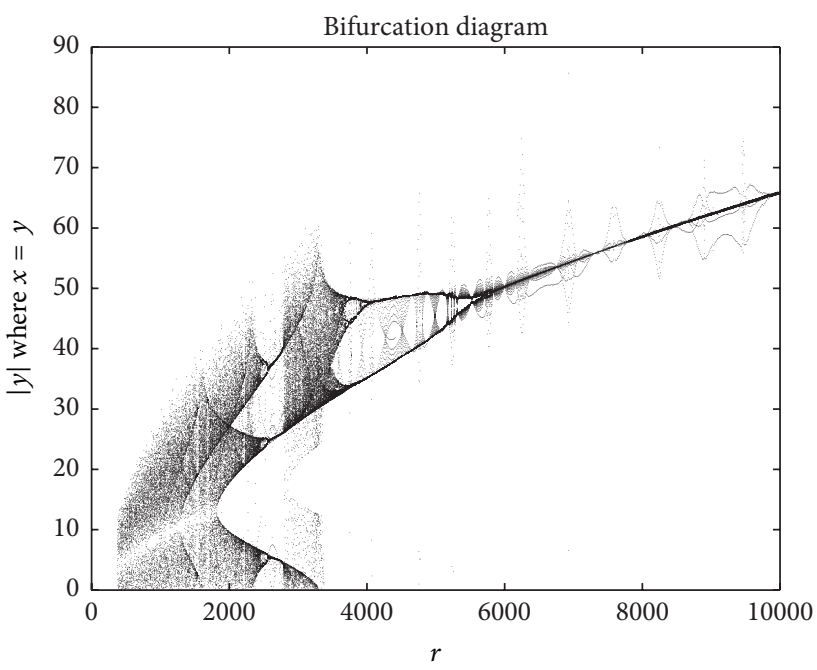

FIGURE 11: The bifurcation diagram of system (1).

now we prove (9) by contradiction. Otherwise, suppose that the trajectory of system (1) stays outside the set $\Omega_{m}$. Since $V_{m}(X)$ is monotonic decreasing outside the set $\Omega_{m}$, the limit

$$
\lim _{t \rightarrow+\infty} V_{m}(X(t))=V_{m}^{*}>\frac{b^{2}(m \sigma+r)^{2}}{2 b-1}
$$

exists. Let

$$
\begin{aligned}
l= & \inf _{X \in D}\left(-\frac{d V_{m}(X)}{d t}\right)\left\{D: V_{m}^{*} \leq m x^{2}(t)+y^{2}(t)\right. \\
& +(z(t)-m \sigma-r)^{2} \leq m x^{2}\left(t_{0}\right)+y^{2}\left(t_{0}\right) \\
& \left.+\left(z\left(t_{0}\right)-m \sigma-r\right)^{2}=V_{m}\left(X\left(t_{0}\right)\right)\right\},
\end{aligned}
$$

where $l>0, V_{m}^{*}>0$ are constants. So we have

$$
\frac{d V_{m}(X)}{d t} \leq-l
$$

Thus, we have $0 \leq V_{m}(X(t))<V_{m}\left(X\left(t_{0}\right)\right)-l\left(t-t_{0}\right) \rightarrow-\infty$ for $t \rightarrow+\infty$. This contradiction implies that expression (9) is true.

From Definition 1, we confirm that $\Omega_{m}$ is a globally attractive set and positively invariant set of system (1). 


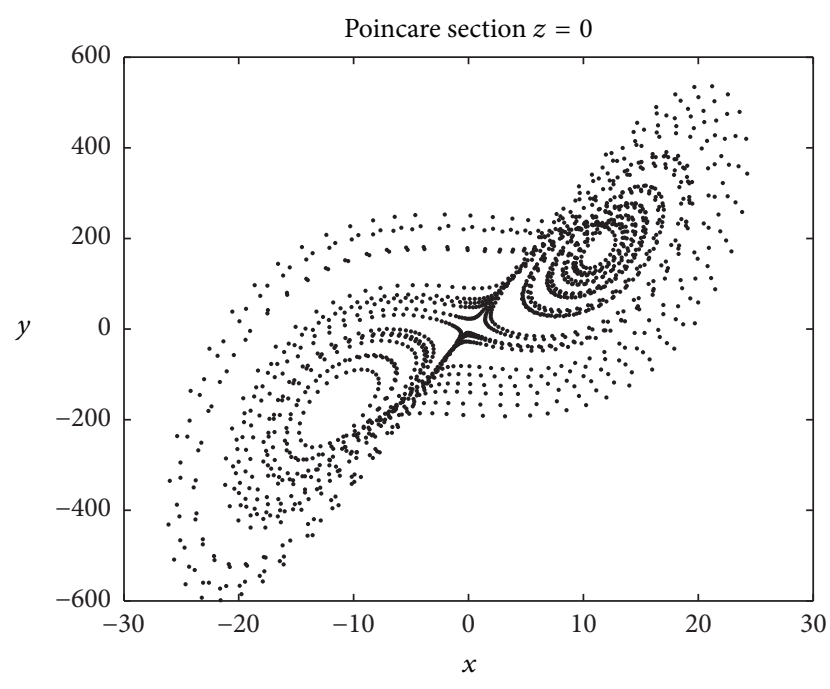

FIgURe 12: The Poincare section of system (1).

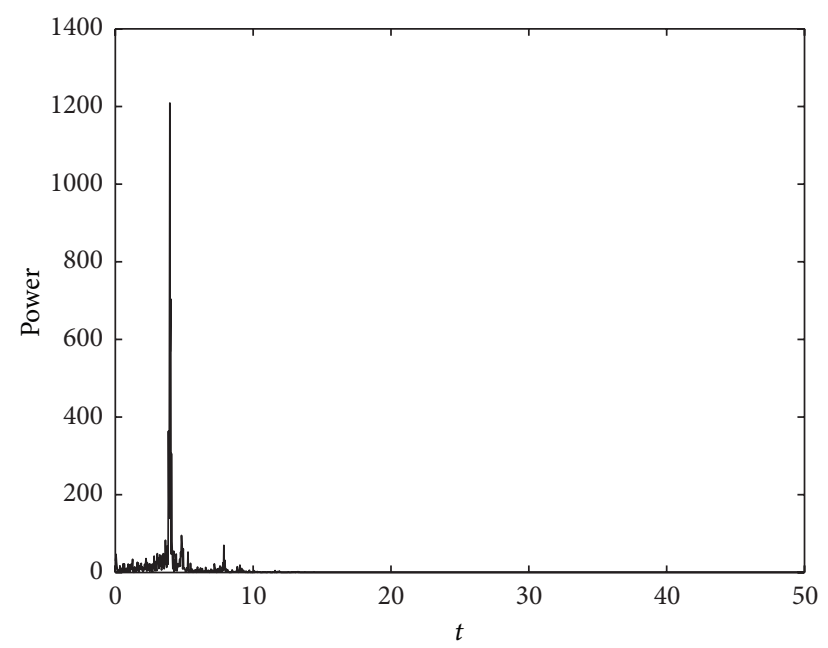

FIGURE 13: The power spectrum of system (1).

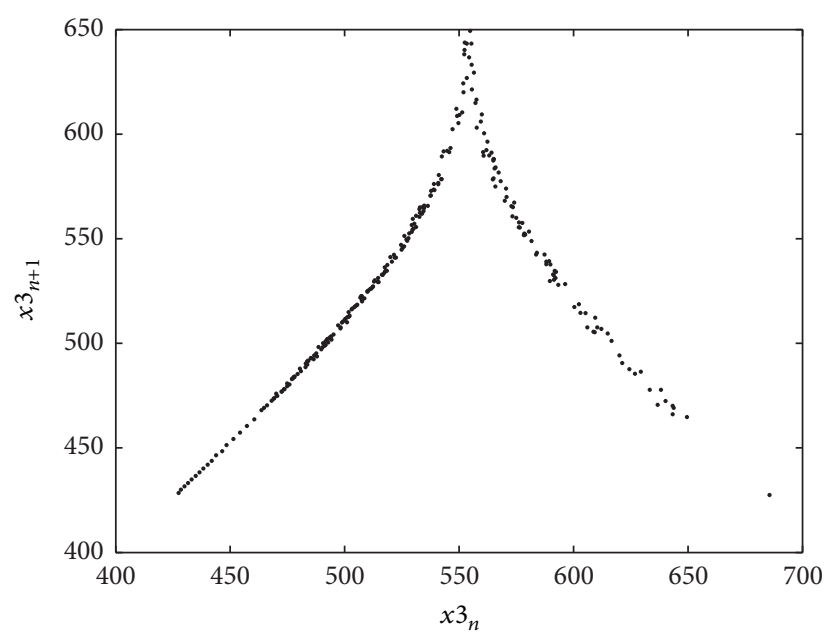

FIgURE 14: The return mapping of system (1).
Remark 3. (1) Taking $m=0$, we get a new cylindrate formula for system (1) as

$$
y^{2}+(z-r)^{2} \leq \frac{b^{2} r^{2}}{2 b-1} .
$$

(2) Taking $m=1$, we get a new ellipsoidal formula for system (1) as

$$
x^{2}+y^{2}+(z-\sigma-r)^{2} \leq \frac{b^{2}(\sigma+r)^{2}}{2 b-1} .
$$

(3) Taking $m=1 / \sigma$, we get another new ellipsoidal formula for system (1) as

$$
\frac{1}{\sigma} x^{2}+y^{2}+(z-1-r)^{2} \leq \frac{b^{2}(1+r)^{2}}{2 b-1} .
$$

(4) Taking $m=r / \sigma$, we get another ellipsoidal formula for system (1) as

$$
\frac{r}{\sigma} x^{2}+y^{2}+(z-2 r)^{2} \leq \frac{4 b^{2} r^{2}}{2 b-1}
$$

Similarly, we can get more estimation formulas by taking different value of $m$.

When $0<\sigma \leq 1$, our estimation (14) is more accurate than estimation (15) with respect to $y$ and $z$. In fact, we can observe the result from the following inequalities:

$$
\begin{aligned}
&|y| \leq \frac{b^{2}(\sigma+r)^{2}}{2 b-1} \leq \frac{b^{2}(1+r)^{2}}{2 b-1}, \\
&(1+r)\left(1-\frac{b}{\sqrt{2 b-1}}\right) \leq(\sigma+r)\left(1-\frac{b}{\sqrt{2 b-1}}\right) \leq z \\
& \quad \leq(\sigma+r)\left(1+\frac{b}{\sqrt{2 b-1}}\right) \\
& \leq(1+r)\left(1+\frac{b}{\sqrt{2 b-1}}\right) .
\end{aligned}
$$

On the other hand, when $\sigma \neq r$, from the following inequality, we can see that our estimation (16) is better than estimation (14), with respect to $x$ :

$$
x^{2} \leq \frac{4 b^{2} \sigma r}{2 b-1} \leq \frac{b^{2}(\sigma+r)^{2}}{2 b-1} .
$$

When $\sigma=r$, for the variable $x$, the estimation (16) is equal to estimation (14).

From the intersection of set theory, we construct different globally attractive sets to get effective results.

Theorem 4. Suppose $\sigma>0, s \geq 10, b>0, r \geq(\sigma+s) / \sigma$. The cylinder given by

$$
\begin{aligned}
& Q_{1}:\left\{\begin{array}{l}
y^{2}+(z-r)^{2} \leq \tau r^{2}, \\
x^{2} \leq \tau r^{2}\left(\frac{\sigma}{\sigma+s}\right)^{2},
\end{array} \quad(\text { when } 0<b \leq 1)\right. \\
& Q_{2}:\left\{\begin{array}{l}
y^{2}+(z-r)^{2} \leq \frac{b^{2} r^{2}}{2 b-1}, \\
x^{2} \leq \frac{b^{2} r^{2}}{2 b-1}\left(\frac{\sigma}{\sigma+s}\right)^{2}
\end{array} \quad(\text { when } b>1)\right.
\end{aligned}
$$


is a globally attractive set and positively invariant set of system (1), where $\tau>1$ is any constant.

Proof. At first, we consider the case of $0<b \leq 1$. For the second and third equations of system (1), we construct a generalized positive definite and radical unbounded Lyapunov function

$$
V=\frac{1}{2}\left(y^{2}+(z-r)^{2}\right) .
$$

We compute the derivate of $V$ along the trajectory of system (1) as

$$
\begin{aligned}
\dot{V} & =y \cdot \dot{y}+(z-r) \dot{z} \\
& =y \cdot(r x-y-x z)+(z-r)(x y-b z) \\
& =-y^{2}-b z^{2}+r b z \leq-y^{2}-\frac{b}{2} z^{2}+\frac{b}{2} \cdot 2 \cdot r z \\
& =-y^{2}-\frac{b}{2}(z-r)^{2}+\frac{b}{2} r^{2} \\
& \leq-\frac{b}{2} y^{2}-\frac{b}{2}(z-r)^{2}+\frac{b}{2} r^{2}<0,
\end{aligned}
$$

for $y^{2}+(z-r)^{2}>r^{2}$. Thus the cylinder $y^{2}+(z-r)^{2} \leq \tau r^{2}$ is a globally attractive set and positively invariant set of system (1) with respect to $y$ and $z$. Then we have $|y| \leq \sqrt{\tau} r$.

Substituting the ultimate boundedness of $y$ into the first equation of system (1) and employing constant variation formula, we have

$$
\begin{aligned}
x(t)= & x\left(t_{0}\right) \cdot e^{-(\sigma+s)\left(t-t_{0}\right)}+\int_{t_{0}}^{t} \sigma \cdot y(\omega) \\
& \cdot e^{-(\sigma+s)(t-\omega)} d \omega \\
\leq & x\left(t_{0}\right) \cdot e^{-(\sigma+s)\left(t-t_{0}\right)}+\int_{t_{0}}^{t} \sigma \cdot \sqrt{\tau} \cdot r \\
& \cdot e^{-(\sigma+s)(t-\omega)} d \omega \\
= & x\left(t_{0}\right) \cdot e^{-(\sigma+s)\left(t-t_{0}\right)}+\sqrt{\tau} \cdot r \\
& \cdot \frac{\sigma}{\sigma+s}\left(1-e^{-(\sigma+s)\left(t-t_{0}\right)}\right) \\
= & \left(x\left(t_{0}\right)-\sqrt{\tau} \cdot r \cdot \frac{\sigma}{\sigma+s}\right) \cdot e^{-(\sigma+s)\left(t-t_{0}\right)}+\sqrt{\tau} \cdot r \\
& \cdot \frac{\sigma}{\sigma+s} .
\end{aligned}
$$

Therefore,

$$
\begin{aligned}
& \left(x(t)-\sqrt{\tau} \cdot r \cdot \frac{\sigma}{\sigma+s}\right) \\
& \quad \leq\left(x\left(t_{0}\right)-\sqrt{\tau} \cdot r \cdot \frac{\sigma}{\sigma+s}\right) \cdot e^{-(\sigma+s)\left(t-t_{0}\right)} \longrightarrow 0,
\end{aligned}
$$

for $x\left(t_{0}\right)>\sqrt{\tau} \cdot r \cdot(\sigma /(\sigma+s)), t \rightarrow+\infty$; that is, $x(t)$ is greater than $\sqrt{\tau} \cdot r \cdot(\sigma /(\sigma+s))$ and tends to $\sqrt{\tau} \cdot r \cdot(\sigma /(\sigma+s))$ exponentially.
At the same time, we have

$$
\begin{aligned}
x(t)= & x\left(t_{0}\right) \cdot e^{-(\sigma+s)\left(t-t_{0}\right)} \\
& +\int_{t_{0}}^{t} \sigma \cdot y(\omega) \cdot e^{-(\sigma+s)(t-\omega)} d \omega \\
\geq & x\left(t_{0}\right) \cdot e^{-(\sigma+s)\left(t-t_{0}\right)}-\sigma \cdot \sqrt{\tau} \cdot r \cdot e^{-(\sigma+s) t} \\
& \cdot \int_{t_{0}}^{t} e^{(\sigma+s) \omega} d \omega \\
= & x\left(t_{0}\right) \cdot e^{-(\sigma+s)\left(t-t_{0}\right)}-\sqrt{\tau} \cdot r \\
& \cdot \frac{\sigma}{\sigma+s}\left(1-e^{-(\sigma+s)\left(t-t_{0}\right)}\right) \\
= & \left(x\left(t_{0}\right)+\sqrt{\tau} \cdot r \cdot \frac{\sigma}{\sigma+s}\right) \cdot e^{-(\sigma+s)\left(t-t_{0}\right)}-\sqrt{\tau} \cdot r \\
& \cdot \frac{\sigma}{\sigma+s} .
\end{aligned}
$$

Thus, one can see that $-x(t)-\sqrt{\tau} \cdot r \cdot(\sigma /(\sigma+s)) \leq-\left(x\left(t_{0}\right)+\sqrt{\tau}\right.$. $r \cdot(\sigma /(\sigma+s))) \cdot e^{-(\sigma+s)\left(t-t_{0}\right)} \rightarrow 0$, for $x\left(t_{0}\right)<-\sqrt{\tau} \cdot r \cdot(\sigma /(\sigma+s))$. That is, $x(t)$ is less than $-\sqrt{\tau} \cdot r \cdot(\sigma /(\sigma+s))$ and tends to $-\sqrt{\tau} \cdot r \cdot(\sigma /((\sigma+s))$ exponentially. Consequently, the set $|x| \leq$ $\sqrt{\tau} \cdot r \cdot(\sigma /(\sigma+s))$ is the globally attractive set and positively invariant set of system (1).

Consider the case of $b>1$. Employing Lyapunov function (20) again, we compute the derivate of $V$ along the trajectory of system (1) as

$$
\begin{aligned}
\dot{V} & =-y^{2}-b z^{2}+r b z \\
& =-V-\frac{1}{2} y^{2}-\left(b-\frac{1}{2}\right) z^{2}+r(b-1) z+\frac{1}{2} r^{2} \\
& =-V+G(X),
\end{aligned}
$$

where $X=(y, z)$, and define the function

$$
G(X)=-\frac{1}{2} y^{2}-\left(b-\frac{1}{2}\right) z^{2}+r(b-1) z+\frac{1}{2} r^{2} .
$$

Computing the extreme value of Lagrange of function $G(X)$ on $(y, z)$, because $G(X)$ is the quadratic function and the local maxima are the global maximum, and letting

$$
\begin{aligned}
& \frac{\partial G}{\partial y}=-y=0 \\
& \frac{\partial G}{\partial z}=-2\left(b-\frac{1}{2}\right) z+r(b-1)=0,
\end{aligned}
$$

we have $y=0, z=r(b-1) /(2 b-1)$.

Computing the second partial derivative of $G(X)$,

$$
\begin{aligned}
& \frac{\partial^{2} G}{\partial y^{2}}=-1<0, \\
& \frac{\partial^{2} G}{\partial x^{2}}=-2\left(b-\frac{1}{2}\right)<0, \quad\left(\text { For } b>\frac{1}{2}\right) \\
& \frac{\partial^{2} G}{\partial y \partial z}=0 ;
\end{aligned}
$$


therefore,

$$
\sup _{X \in R^{2}} G(X)=\left.G(y, z)\right|_{(y=0, z=r(b-1) /(2 b-1))}=\frac{b^{2} r^{2}}{2(2 b-1)} .
$$

When $V>b^{2} r^{2} / 2(2 b-1), \dot{V}<0$. Now we prove by contradiction similar to Theorem 2 that $\lim _{t \rightarrow+\infty} \rho\left(X\left(t, t_{0}, X_{0}\right), Q_{2}\right)=$ 0 , so the set $y^{2}+(z-r)^{2} \leq b^{2} r^{2} /(2 b-1)$ is the globally attractive set and positively invariant set of system (1).

From

$$
y^{2}+(z-r)^{2} \leq \frac{b^{2} r^{2}}{2 b-1},
$$

we have $|y| \leq b r / \sqrt{2 b-1}$. Similar to (23) and (24), we can get $|x(t)| \leq(b r / \sqrt{2 b-1}) \cdot(\sigma /(\sigma+s))$, so the set $|x(t)| \leq$ $(b r / \sqrt{2 b-1}) \cdot(\sigma /(\sigma+s))$ is the globally attractive set with respect to $x$ that shows that $Q_{2}$ is globally attractive set and positively invariant set of system (1).

\section{Applications to Global and Exponential Tracking (or Stabilizing) of Any $\omega$-Period Solution}

There are two aims of the chaotic control. One is to stabilize an unstable (or stable but not asymptotically stable) equilibrium so that it can become a globally asymptotically stable or even a globally exponentially stable one. The other is to track a special unstable $\omega$-period solution, that is, to control all the solutions and let them converge to this $\omega$-period solution. Due to existence of the globally attractive set, all the solutions will enter this globally attractive compact set. In the compact set, the right side of system (1) must satisfy Lipschitz condition. This fact ensures the existence of some linear feedback controllers so that the controlled system can track some $\omega$-period solution or can be stabilized.

Suppose that $X^{*}(t)=\left(x^{*}(t), y^{*}(t), z^{*}(t)\right)^{T}$ is any periodic solution or any equilibrium of system (1). Let $\bar{X}(t)=$ $X(t)-X^{*}(t)$. Then $\bar{X}(t)$ satisfies

$$
\begin{aligned}
& \dot{\bar{x}}=-(\sigma+s) \bar{x}+\sigma \bar{y}, \\
& \dot{\bar{y}}=r \bar{x}-\bar{y}-x z+x^{*} z^{*}, \\
& \dot{\bar{z}}=x y-x^{*} y^{*}-b \bar{z} .
\end{aligned}
$$

System (31) with controllers can be written as

$$
\begin{aligned}
& \dot{\bar{x}}=-(\sigma+s) \bar{x}+\sigma \bar{y}-k_{1} \bar{x}, \\
& \dot{\bar{y}}=r \bar{x}-\bar{y}-x z+x^{*} z^{*}-k_{2} \bar{y}, \\
& \dot{\bar{z}}=x y-x^{*} y^{*}-b \bar{z}-k_{3} \bar{z} .
\end{aligned}
$$

Now we design some simplest linear feedback controllers so that the zero solution of the controlled system (32) is globally exponentially stable. When $X^{*}(t)$ is a periodic solution, we will confirm that all the solutions of system (1) track $X^{*}(t)$ globally and exponentially, and when $X^{*}(t)$ is an equilibrium $X^{*}$, we will prove that $X^{*}$ can be globally exponentially stabilized.
Theorem 5. The control parameters always can be selected as $k_{2}=k_{3}=0$ and $k_{1}>0$ suitably large so that the zero solution of system (32) is globally exponentially stable. In other words, all the solutions of system (1) track the periodic solution $X^{*}(t)$ globally and exponentially, or the equilibrium $X^{*}$ can be globally and exponentially stabilized.

Proof. (1) Suppose that $X^{*}(t)$ is a known periodic solution. Select the parameters as

$$
k_{1}>\frac{y^{* 2}}{4 b}+\frac{1}{4}\left(\sigma+r-z^{*}\right)^{2}-(\sigma+s) .
$$

It implies that the following matrix of periodic functions is negative definite [20-23]:

$$
G_{1}(t)=\left(\begin{array}{ccc}
-2\left(\sigma+s+k_{1}\right) & \sigma+r-z^{*} & y^{*} \\
\sigma+r-z^{*} & -2 & 0 \\
y^{*} & 0 & -2 b
\end{array}\right) .
$$

Denote by $\bar{\lambda}_{1}(t)\left(G_{1}\right)$ the maximal eigenvalues of $G_{1}(t)$. Then, we have $-\beta=\max _{t \in(0, \omega)} \bar{\lambda}_{1}(t)\left(G_{1}\right)(\beta>0)$.

Construct a positive definite and radical unbounded Lyapunov function for system (32) as

$$
V(\bar{X})=\bar{x}^{2}+\bar{y}^{2}+\bar{z}^{2}
$$

We compute the derivative of it and have

$$
\begin{aligned}
& \frac{d V}{d t}=2 \bar{x} \cdot \dot{\bar{x}}+2 \bar{y} \cdot \dot{\bar{y}}+2 \bar{z} \cdot \dot{\bar{z}}=2 \bar{x} \\
& \cdot\left(-(\sigma+s) \bar{x}+\sigma \bar{y}-k_{1} \bar{x}\right)+2 \bar{y} \\
& \cdot\left(r \bar{x}-\bar{y}-x z+x^{*} z^{*}\right)+2 \bar{z}\left(x y-x^{*} y^{*}-b \bar{z}\right) \\
& =-2(\sigma+s) \bar{x}^{2}+2 \sigma \overline{x y}-2 k_{1} \bar{x}^{2}+2 r \overline{x y}-2 \bar{y}^{2} \\
& -2 z^{*} \overline{x y}+2 y^{*} \overline{x z}-2 b \bar{z}^{2}=\left(\begin{array}{c}
\bar{x} \\
\bar{y} \\
\bar{z}
\end{array}\right)^{T} \\
& \cdot\left(\begin{array}{ccc}
-2\left(\sigma+s+k_{1}\right) & \sigma+r-z^{*} & y^{*} \\
\sigma+r-z^{*} & -2 & 0 \\
y^{*} & 0 & -2 b
\end{array}\right)\left(\begin{array}{c}
\bar{x} \\
\bar{y} \\
\bar{z}
\end{array}\right) \\
& \leq \bar{\lambda}_{1}(t)\left(G_{1}\right)\left(\bar{x}^{2}(t)+\bar{y}^{2}(t)+\bar{z}^{2}(t)\right) \text {. }
\end{aligned}
$$

According to Gronwall inequation, formula (36) is transformed as

$$
\begin{aligned}
& \left(\bar{x}^{2}(t)+\bar{y}^{2}(t)+\bar{z}^{2}(t)\right) \\
& \quad \leq\left(\bar{x}^{2}\left(t_{0}\right)+\bar{y}^{2}\left(t_{0}\right)+\bar{z}^{2}\left(t_{0}\right)\right) e^{\int_{t_{0}}^{t} \bar{\lambda}_{1}(s) d s}, \quad t \geq t_{0} .
\end{aligned}
$$

Formula (37) implies the conclusion.

(2) Suppose that the expression of $X^{*}(t)$ is unknown and the amplitude of it is known or can be estimated. 
Suppose $x_{g}=\max _{t \in(0, \omega)}|x(t)|, y_{g}=\max _{t \in(0, \omega)}|y(t)|$, $z_{g}=\max _{t \in(0, \omega)}|z(t)|$ are amplitudes of the 3 components of the periodic solution. The parameters can be selected as

$$
k_{1}>\frac{y_{g}^{2}}{4 b}+\frac{1}{4}\left(\sigma+r+z_{g}\right)^{2}-(\sigma+s) \text {. }
$$

It implies that the following constant matrix is negative definite:

$$
G_{2}=\left(\begin{array}{ccc}
-2\left(\sigma+s+k_{1}\right) & \sigma+r+z_{g} & y_{g} \\
\sigma+r+z_{g} & -2 & 0 \\
y_{g} & 0 & -2 b
\end{array}\right)
$$

By $\bar{\lambda}_{2}\left(G_{2}\right)$, we denote the maximal eigenvalues of matrix $G_{2}$; then $-\gamma=\max _{t \in(0, \omega)} \bar{\lambda}_{2}\left(G_{2}\right)$. Employing the Lyapunov function (35), we have

$$
\begin{aligned}
& \frac{d V}{d t}=-2\left(\sigma+s+k_{1}\right) \bar{x}^{2}-2 \bar{y}^{2}-2 b \bar{z}^{2} \\
& +2\left(\sigma+r-z^{*}\right) \overline{x y}+2 y^{*} \overline{x z} \leq-2\left(\sigma+s+k_{1}\right)|\bar{x}|^{2} \\
& -2|\bar{y}|^{2}-2 b|\bar{z}|^{2}+2\left(\sigma+r+z_{g}\right)|\bar{x}||\bar{y}|+2 y_{g}|\bar{x}| \\
& \cdot|\bar{z}|=\left(\begin{array}{c}
|\bar{x}| \\
|\bar{y}| \\
|\bar{z}|
\end{array}\right) \\
& \quad\left(\begin{array}{ccc}
-2\left(\sigma+s+k_{1}\right) & \sigma+r+z_{g} & y_{g} \\
\sigma+r+z_{g} & -2 & 0 \\
y_{g} & 0 & -2 b
\end{array}\right)\left(\begin{array}{c}
|\bar{x}| \\
|\bar{y}| \\
|\bar{z}|
\end{array}\right) \\
& \quad\left(\begin{array}{cc}
\bar{\lambda}_{2}\left(G_{2}\right)\left(x^{2}+y^{2}+z^{2}\right) . &
\end{array}\right.
\end{aligned}
$$

Hence, we have

$$
\begin{aligned}
& \left(\bar{x}^{2}(t)+\bar{y}^{2}(t)+\bar{z}^{2}(t)\right) \\
& \quad \leq\left(\bar{x}^{2}\left(t_{0}\right)+\bar{y}^{2}\left(t_{0}\right)+\bar{z}^{2}\left(t_{0}\right)\right) e^{-\gamma\left(t-t_{0}\right)}, \quad t \geq t_{0} .
\end{aligned}
$$

This inequality implies the conclusion.

(3) Suppose the amplitude of $X^{*}(t)$ is unknown. The periodic solution is assumed to be in the globally attractive set. We can use the boundedness of globally attractive set as the boundedness of $X^{*}(t)$.

If $b>1$, we select $k_{2}=k_{3}=0$ and

$$
k_{1}>\frac{b r^{2}}{4(2 b-1)}+\frac{1}{4}\left(\sigma+\frac{b r}{\sqrt{2 b-1}}\right)^{2}-(\sigma+s) \text {. }
$$

This guarantees that the following constant matrix is negative definite:

$$
=\left(\begin{array}{ccc}
-2\left(\sigma+s+k_{1}\right) & \sigma+\frac{b r}{\sqrt{2 b-1}} & \frac{b r}{\sqrt{2 b-1}} \\
\sigma+\frac{b r}{\sqrt{2 b-1}} & -2 & 0 \\
\frac{b r}{\sqrt{2 b-1}} & 0 & -2 b
\end{array}\right) \text {. }
$$

Denote by $\bar{\lambda}_{3}\left(G_{3}\right)$ the maximal eigenvalues of matrix $G_{3}$. Employing the Lyapunov function (35), we have

$$
\begin{aligned}
& \frac{d V}{d t}=\left(\begin{array}{c}
\bar{x} \\
\bar{y} \\
\bar{z}
\end{array}\right)^{T}\left(\begin{array}{ccc}
-2\left(\sigma+s+k_{1}\right) & \sigma+r-z^{*} & y^{*} \\
\sigma+r-z^{*} & -2 & 0 \\
y^{*} & 0 & -2 b
\end{array}\right)\left(\begin{array}{c}
\bar{x} \\
\bar{y} \\
\bar{z}
\end{array}\right) \\
& \leq\left(\begin{array}{c}
|\bar{x}| \\
|\bar{y}| \\
|\bar{z}|
\end{array}\right)^{T} \\
& \cdot\left(\begin{array}{ccc}
-2\left(\sigma+s+k_{1}\right) & \sigma+\frac{b r}{\sqrt{2 b-1}} & \frac{b r}{\sqrt{2 b-1}} \\
\sigma+\frac{b r}{\sqrt{2 b-1}} & -2 & 0 \\
\frac{b r}{\sqrt{2 b-1}} & 0 & -2 b
\end{array}\right)\left(\begin{array}{c}
|\bar{x}| \\
|\bar{y}| \\
|\bar{z}|
\end{array}\right) \\
& \leq \bar{\lambda}_{3}\left(G_{3}\right)\left(\bar{x}^{2}+\bar{y}^{2}+\bar{z}^{2}\right) \text {. }
\end{aligned}
$$

Hence, we obtain

$$
\bar{x}^{2}+\bar{y}^{2}+\bar{z}^{2} \leq V\left(X\left(t_{0}\right)\right) e^{\bar{\lambda}_{3}\left(G_{3}\right)\left(t-t_{0}\right)}, \quad t \geq t_{0} .
$$

This ensures the conclusion.

When $0<b \leq 1$, we take

$$
\begin{aligned}
& k_{2}=k_{3}=0, \\
& k_{1}>\frac{\tau r^{2}}{4 b}+\frac{1}{4}(\sigma+\sqrt{\tau} r)^{2}-(\sigma+s) .
\end{aligned}
$$

It ensures that the following constant matrix is negative definite:

$$
G_{4}=\left(\begin{array}{ccc}
-2\left(\sigma+s+k_{1}\right) & \sigma+\sqrt{\tau} r & \sqrt{\tau} r \\
\sigma+\sqrt{\tau} r & -2 & 0 \\
\sqrt{\tau} r & 0 & -2 b
\end{array}\right) .
$$


Denote by $\bar{\lambda}_{4}\left(G_{4}\right)$ the matrix eigenvalues of matrix $G_{4}$. Employing the Lyapunov function (35), we have

$$
\begin{aligned}
\frac{d V}{d t} & =\left(\begin{array}{c}
\bar{x} \\
\bar{y} \\
\bar{z}
\end{array}\right)^{T} \\
& \cdot\left(\begin{array}{ccc}
-2\left(\sigma+s+k_{1}\right) & \sigma+r-z^{*} & y^{*} \\
\sigma+r-z^{*} & -2 & 0 \\
y^{*} & 0 & -2 b
\end{array}\right)\left(\begin{array}{c}
\bar{x} \\
\bar{y} \\
\bar{z}
\end{array}\right) \\
& \leq\left(\begin{array}{ccc}
|\bar{x}| \\
|\bar{y}| \\
|\bar{z}|
\end{array}\right)^{T} \\
& \left(\begin{array}{ccc}
-2\left(\sigma+s+k_{1}\right) & \sigma+\sqrt{\tau} r & \sqrt{\tau} r \\
\sigma+\sqrt{\tau} r & -2 & 0 \\
\sqrt{\tau} r & 0 & -2 b
\end{array}\right)\left(\begin{array}{c}
|\bar{x}| \\
|\bar{y}| \\
|\bar{z}|
\end{array}\right) \\
& \leq \bar{\lambda}_{4}\left(G_{4}\right)\left(\bar{x}^{2}+\bar{y}^{2}+\bar{z}^{2}\right) .
\end{aligned}
$$

Hence, we obtain

$$
\bar{x}^{2}+\bar{y}^{2}+\bar{z}^{2} \leq V\left(X\left(t_{0}\right)\right) e^{\bar{\lambda}_{4}\left(G_{4}\right)\left(t-t_{0}\right)}, \quad t \geq t_{0} .
$$

This ensures the conclusion. The proof of Theorem 5 is complete.

\section{Globally Exponential Synchronization of Two Chaotic Systems}

Definition 6. Two nonlinear dynamic systems [24] are given as follows:

$$
\begin{aligned}
& \dot{X}=F(t, X), \\
& \dot{Y}=F(t, Y)+\mu(X, Y),
\end{aligned}
$$

where $X, Y \in R^{n}$ and the function $F$ is an $n$-dimensional nonlinear function. Function $\mu$ is an $n$-dimensional control input function. We call system (50) the drive system and system (51) the response system. If $\lim _{t \rightarrow \infty}\|Y(t)-X(t)\|=0$, then system (50) and system (51) are exponentially synchronized.

Consider two similar systems of system (1); the variables of the drive system are marked with the subscript 1 and the variables of the response system are marked with the subscript 2.

Suppose that the drive system is

$$
\begin{aligned}
& \dot{x}_{1}=-(\sigma+s) x_{1}+\sigma y_{1}, \\
& \dot{y}_{1}=r x_{1}-y_{1}-x_{1} z_{1}, \\
& \dot{z}_{1}=x_{1} y_{1}-b z_{1} .
\end{aligned}
$$

The response system is

$$
\begin{aligned}
& \dot{x}_{2}=-(\sigma+s) x_{2}+\sigma y_{2}+\mu_{1}\left(e_{x}, e_{y}, e_{z}\right), \\
& \dot{y}_{2}=r x_{2}-y_{2}-x_{2} z_{2}+\mu_{2}\left(e_{x}, e_{y}, e_{z}\right), \\
& \dot{z}_{2}=x_{2} y_{2}-b z_{2}+\mu_{3}\left(e_{x}, e_{y}, e_{z}\right),
\end{aligned}
$$

where $\mu_{1}, \mu_{2}$, and $\mu_{3}$ are the controllers designed satisfying $\mu_{i}(0,0,0)=0, i=1,2,3$.

Let $e^{T}=\left(e_{x}, e_{y}, e_{z}\right), e_{x}=x_{2}-x_{1}, e_{y}=y_{2}-y_{1}, e_{z}=$ $z_{2}-z_{1}$; then we have the error dynamic system as follows:

$$
\begin{aligned}
& \dot{e}_{x}=-(\sigma+s) e_{x}+\sigma e_{y}+\mu_{1}, \\
& \dot{e}_{y}=r e_{x}-e_{y}-z_{2} e_{x}-x_{2} e_{z}+e_{x} e_{z}+\mu_{2}, \\
& \dot{e}_{z}=y_{2} e_{x}+x_{2} e_{y}-e_{x} e_{y}-b e_{z}+\mu_{3} .
\end{aligned}
$$

The aim is to design the effective controllers $\mu_{1}, \mu_{2}$, and $\mu_{3}$ to ensure that the zero solution of the error dynamic system (54) is globally exponentially stable. It means that the drive system (52) and the response system (53) are globally exponentially synchronized. That is to say that $\lim _{t \rightarrow \infty}\|e(t)\|=0$.

We suppose that $|x| \leq M_{x},|y| \leq M_{y},|z| \leq M_{z}$ because the chaotic system is bounded.

Definition 7. If there exists constant $\alpha>0$ satisfying $V(t) \leq$ $V\left(t_{0}\right) e^{-\alpha\left(t-t_{0}\right)}$ for arbitrary $t>t_{0}$, then the origin of the system is said to be exponentially stable.

Theorem 8. For the error dynamic system (54), the controller is designed as follows:

$$
\begin{aligned}
& \mu_{1}=-\sigma e_{y}-k e_{x}, \\
& \mu_{2}=z_{2} e_{x}-r e_{x}, \\
& \mu_{3}=-y_{2} e_{x} .
\end{aligned}
$$

We can select the parameter $k>0$ to make the matrix

$$
P=\left(\begin{array}{ccc}
\sigma+s+k & 0 & 0 \\
0 & 1 & 0 \\
0 & 0 & b
\end{array}\right)
$$

positive definite. Then the zero solution of the error dynamic system (54) is globally exponentially synchronized. It means that the drive system (52) and the response system (53) are globally exponentially synchronized.

Proof. We construct a positive definite and radical unbounded Lyapunov function as

$$
V=\frac{1}{2}\left(e_{x}^{2}+e_{y}^{2}+e_{z}^{2}\right)
$$


Computing the time derivative of $V$ along the positive halftrajectory of system (1), we have

$$
\begin{aligned}
\frac{d V}{d t}= & e_{x} \dot{e}_{x}+e_{y} \dot{e}_{y}+e_{z} \dot{e}_{z} \\
= & e_{x}\left(-(\sigma+s) e_{x}+\sigma e_{y}+\mu_{1}\right) \\
& +e_{y}\left(r e_{x}-e_{y}-z_{2} e_{x}-x_{2} e_{z}+e_{x} e_{z}+\mu_{2}\right) \\
& +e_{z}\left(y_{2} e_{x}+x_{2} e_{y}-e_{x} e_{y}-b e_{z}+\mu_{3}\right) \\
= & -((\sigma+s)+k) e_{x}^{2}-e_{y}^{2}-b e_{z}^{2} \\
= & \left(\begin{array}{c}
e_{x} \\
e_{y} \\
e_{z}
\end{array}\right)^{T}\left(\begin{array}{ccc}
-(\sigma+s+k) & 0 & 0 \\
0 & -1 & 0 \\
0 & 0 & -b
\end{array}\right)\left(\begin{array}{l}
e_{x} \\
e_{y} \\
e_{z}
\end{array}\right) \\
= & -\left(\begin{array}{ccc}
e_{x} \\
e_{y} \\
e_{z}
\end{array}\right)^{T}\left(\begin{array}{ccc}
\sigma+s+k & 0 & 0 \\
0 & 1 & 0 \\
0 & 0 & b
\end{array}\right)\left(\begin{array}{l}
e_{x} \\
e_{y} \\
e_{z}
\end{array}\right) \\
= & -e^{T} P e,
\end{aligned}
$$

where

$$
P=\left(\begin{array}{ccc}
\sigma+s+k & 0 & 0 \\
0 & 1 & 0 \\
0 & 0 & b
\end{array}\right)
$$

Obviously, the zero solution of the error dynamic system (54) is globally exponentially stable when the matrix $P$ is positive definite under the condition that the following inequalities are established:

$$
\begin{array}{r}
\sigma+s+k>0, \\
b(\sigma+s+k)>0 ;
\end{array}
$$

we can have $k>-(\sigma+s)$.

Thus, when $k>0$, the matrix $P$ is positive definite and $\dot{V}$ is negative definite. It can be observed from formula (58) and the knowledge of advanced algebra that

$$
\frac{d V}{d t} \leq-\lambda_{\min }(P)\left(e_{x}^{2}+e_{y}^{2}+e_{z}^{2}\right) \leq-\lambda_{\min }(P) V .
$$

Therefore,

$$
V(X(t)) \leq V\left(X\left(t_{0}\right)\right) e^{-\lambda_{\min }(P)\left(t-t_{0}\right)}, \quad t \geq t_{0} .
$$

When $t \rightarrow+\infty, V(X(t)) \rightarrow 0$. It ensures that the zero solution of the error dynamic system (54) is globally exponentially stable. It means that the drive system (52) and the response system (53) are globally exponentially synchronized.

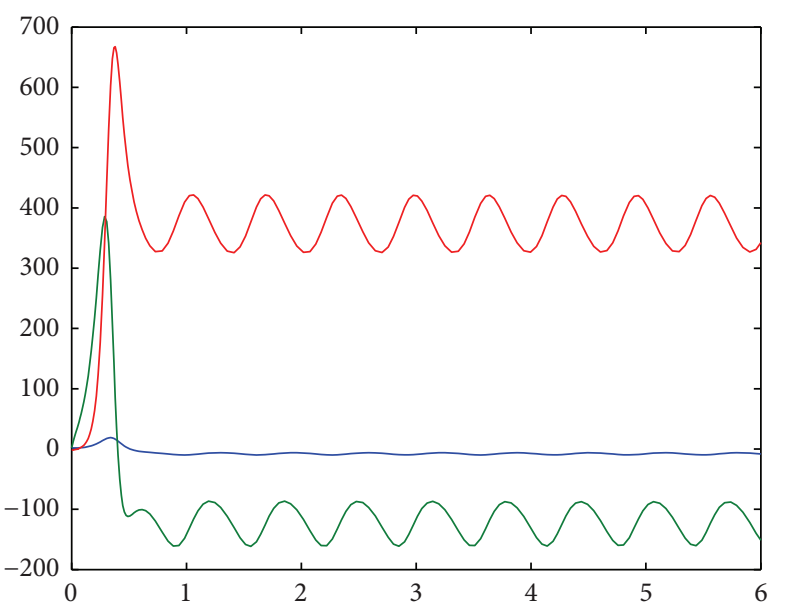

FIGURE 15: State of variants $x, y, z$ of system (1) without control law.

\section{Numerical Simulation}

In this section, we will do some numerical simulations to verify the effectiveness of the proposed methods by using the Runge-Kutta method. In the numerical simulations, the time step size is 0.001 , and the parameters are chosen as $\sigma=0.7$, $s=10, b=8 / 3$ to ensure the existence of system (1).

For the numerical simulation, we consider the systems given as (52) and (53). The initial values of the drive system and response system are taken as $\left(x_{1}(0), y_{1}(0), z_{1}(0)\right)=$ $(0.5,0.9,1.2),\left(x_{2}(0), y_{2}(0), z_{2}(0)\right)=(-0.8,-0.4,-1)$. Hence, the initial error is $\left(e_{x}(0), e_{y}(0), e_{z}(0)\right)=(-1.3,-1.3,-2.2)$.

Considering controller in Theorem 8 , we select the feedback gain $k=1$ as the controller law of system (53) for the linear feedback synchronization. The simulation results are illustrated in Figures 15-17. In Figure 15, we can notice that the state variants $x, y, z$ of system (1) are unstable; this is when there is no controller. Figure 16 displays the time evolution of the drive system and the response system when we add the controller law. From Figure 16, we can see that the two systems starting from different conditions are synchronized with each other. The dynamics of synchronization error is defined as $e(t)=\sqrt{e_{x}^{2}(t)+e_{y}^{2}(t)+e_{z}^{2}(t)}$, whose time evolution is shown in Figure 17. It is clear that the error signals converge to zero finally.

\section{Conclusions}

In this paper, a low mode system in the internal wave dynamics is studied. Dynamical behaviors of the system are analyzed including some basic dynamical properties, such as the attractor diagram, bifurcation diagram, Poincare section, power spectrum, and return mapping, and we obtain the estimation of the globally exponentially attractive set and the positive invariant set of the system via selecting appropriate positive definite and radical unbounded Lyapunov function. On this basis, for any periodic solution, we design some feedback controllers to control all the trajectories of the chaotic system tracking the special periodic solution globally. 

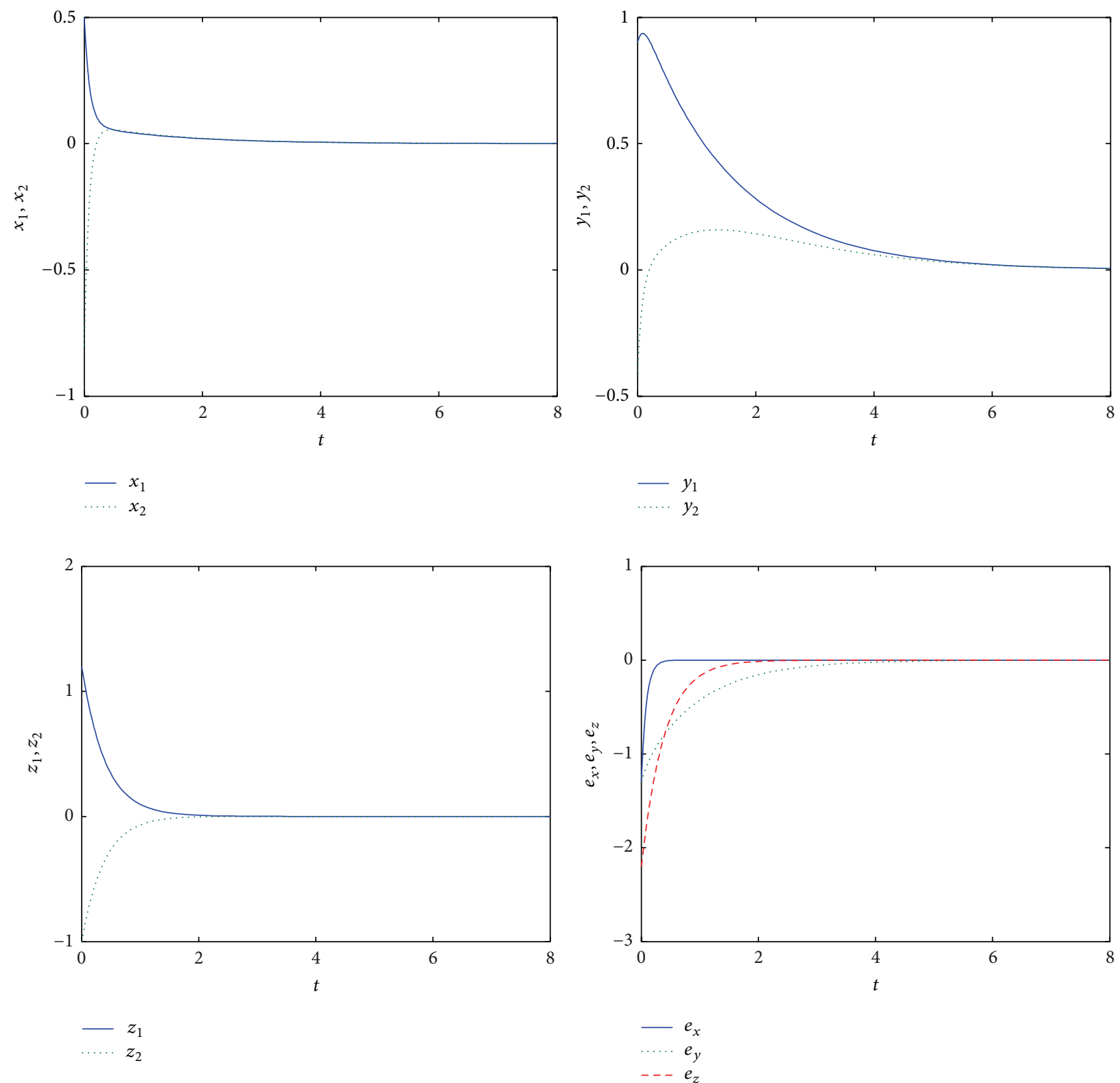

FIGURE 16: Linear feedback synchronization $k=1$.

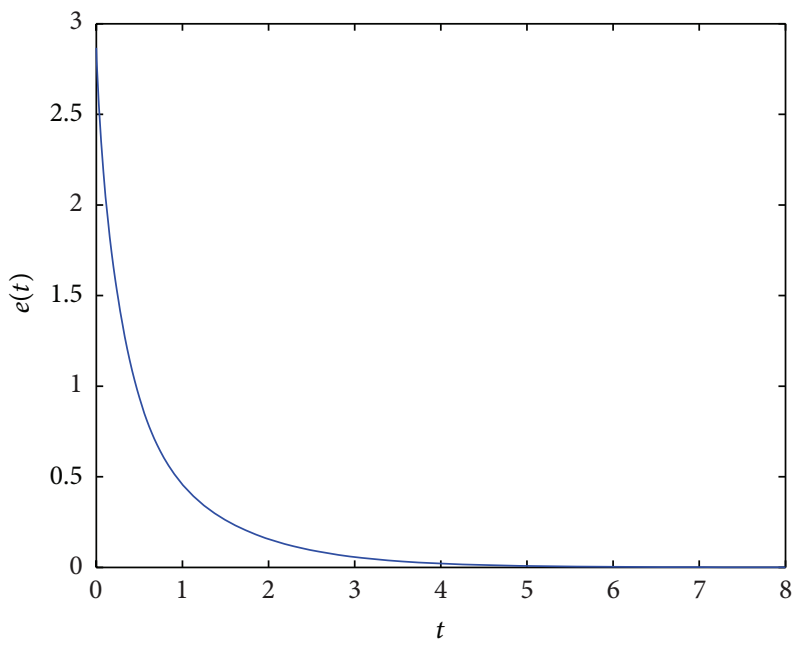

Figure 17: Error $e(t)$ with the change of time $t$. 
The globally exponential synchronization of the chaotic system is realized by the method of drive-response synchronization, and the synchronization conditions are given. The effectiveness of the method is shown by theoretical analysis and numerical simulation.

\section{Competing Interests}

The authors declare that there are no competing interests regarding the publication of this paper.

\section{Acknowledgments}

This research was supported by the National Natural Science Foundation of China under Project nos. 11572146 and 11526105, the Research Fund of Liaoning Provincial Education Department under Project no. L2013248, and the Jinzhou Science and Technology Special Fund under Project no. 13A1D32.

\section{References}

[1] E. N. Lorenz, "Deterministic non-periods flows," Atoms Science, vol. 20, pp. 130-141, 1963.

[2] Y. Shu, Y. Zhang, and H. Xu, "Control and synchronization of general Lorenz chaotic system," Journal of Chongqing Institute of Technology (Natural Sciences), vol. 22, no. 8, pp. 54-61, 2008.

[3] D. G. Thomas, B. Khomami, and R. Sureshkumar, "Nonlinear dynamics of viscoelastic Taylor-Couette flow: effect of elasticity on pattern selection, molecular conformation and drag," Journal of Fluid Mechanics, vol. 620, pp. 353-382, 2009.

[4] W.-S. Kim, "Application of Taylor vortex to crystallization," Journal of Chemical Engineering of Japan, vol. 47, no. 2, pp. 115123, 2014.

[5] H. L. Swinney and J. P. Gollub, Hydrodynamic Instabilities and the Transition to Turbulence, Springer, Berlin, Germany, 1981.

[6] G. I. Taylor, "Stability of a viscons liquid contained between two rotating cylinders," Philosophical Transactions of the Royal Society of London A, vol. 223, pp. 289-343, 1923.

[7] H. Liu and B. Jiang, "Chaos control of gear system with elostomeric web based on multi-parameter multistep method," Journal of Harbin Institute of Technology, vol. 19, no. 5, pp. 23-30, 2012.

[8] G. R. Chen and X. Dong, From Chaos to Order: Methodologies, Perspectives and Applications, World Scientific, Singapore, 1998.

[9] H. L. Swinney and J. P. Gollub, Hydrodynamic, Instablitities and the Transition to Turbulence, Springer, Berlin, Germany, 1981.

[10] H. Y. Wang, "The Chaos behavior and simulation of threemodel systems of Couette-Taylor flow," Acta Mathematica Scientia. Series A, vol. 35, no. 4, pp. 769-779, 2015.

[11] S. Liu, "The wave mechanics in chaos and atmospheric turbulence," Science in China (Series B), vol. 5, 1986.

[12] L. M. Pecora and T. L. Carroll, "Synchronization in chaotic systems," Physical Review Letters, vol. 64, no. 8, pp. 821-824, 1990.

[13] L. M. Pecora and T. L. Carroll, "Driving systems with chaotic signals," Physical Review A, vol. 44, no. 4, pp. 2374-2383, 1991.

[14] D.-Y. Chen, C. Wu, C.-F. Liu, X.-Y. Ma, Y.-J. You, and R.F. Zhang, "Synchronization and circuit simulation of a new double-wing chaos," Nonlinear Dynamics, vol. 67, no. 2, pp. 1481-1504, 2012.

[15] D. Chen, W. Zhao, X. Liu, and X. Ma, "Synchronization and antisynchronization of a class of chaotic systems with nonidentical orders and uncertain parameters," Journal of Computational and Nonlinear Dynamics, vol. 10, no. 1, Article ID 011003, 2015.

[16] J. Guckenheimer, Nonlinear Oscillation, Dynamical Systems, and Bifurcation of Vector Fields, Springer, Berlin, Germany, 1983.

[17] J. H. Ge and J. Xu, "Hopf bifurcation and chaos in an inertial neuron system with coupled delay," Science China Technological Sciences, vol. 56, no. 9, pp. 2299-2309, 2013.

[18] H. A. El-Saka, E. Ahmed, M. I. Shehata, and A. M. A. El-Sayed, "On stability, persistence, and Hopf bifurcation in fractional order dynamical systems," Nonlinear Dynamics, vol. 56, no. 12, pp. 121-126, 2009.

[19] X. Liao, "On the new results of global attractive set and positive invariant set of the Lorenz chaotic system and the applications to chaos control and synchronization," Science in China, Series E: Information Sciences, vol. 34, no. 12, pp. 1404-1419, 2004.

[20] L. Huang, Theory and Application of the Stability and Robustness, National Defend Industy Press, Beijing, China, 2000.

[21] A. A. Martynyuk, "Elements of the theory of stability of hybrid systems (review)," International Applied Mechanics, vol. 51, no. 3, pp. 243-302, 2015.

[22] X. Liao, The Stability Theory and Application of Dynamical System, National Defend Industy Press, Beijing, China, 2000.

[23] V. I. Vorotnikov and Yu. G. Martyshenko, "On partial stability theory of nonlinear dynamic systems," Journal of Computer and Systems Sciences International, vol. 49, no. 5, pp. 702-709, 2010.

[24] J. Fang, "Chaos control method, synchronization principle and its application prospect in nonlinear system (two)," Progress in Physics, vol. 16, no. 2, pp. 137-159, 1996. 


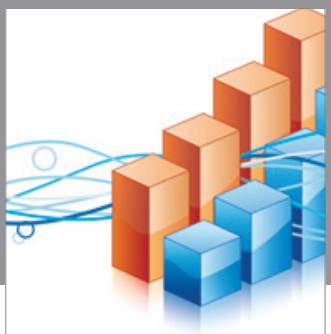

Advances in

Operations Research

vatem alat4

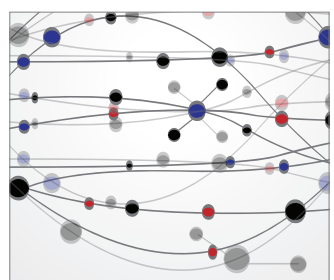

\section{The Scientific} World Journal
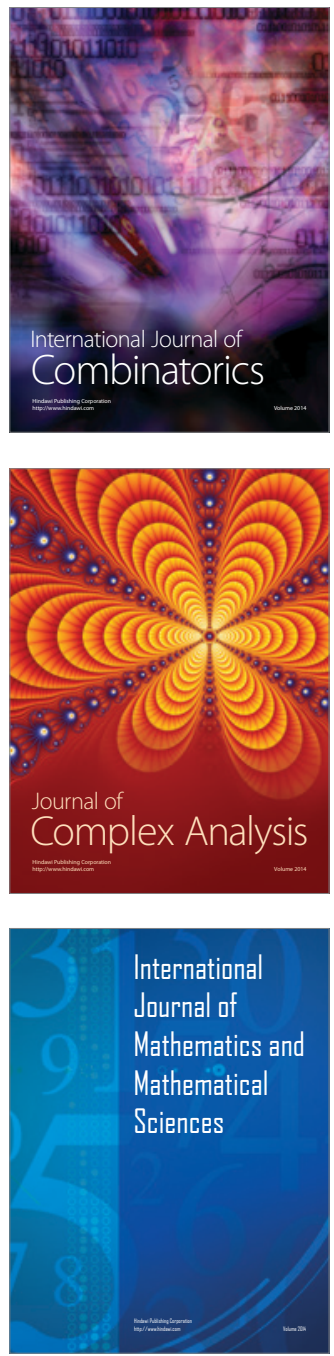
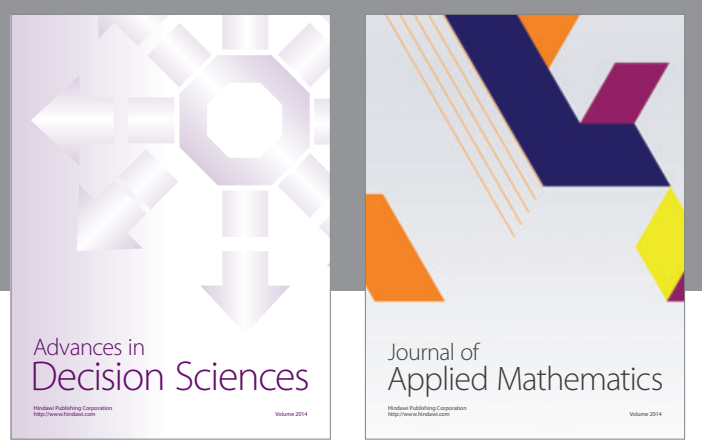

Algebra

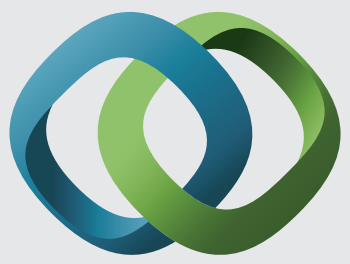

\section{Hindawi}

Submit your manuscripts at

http://www.hindawi.com
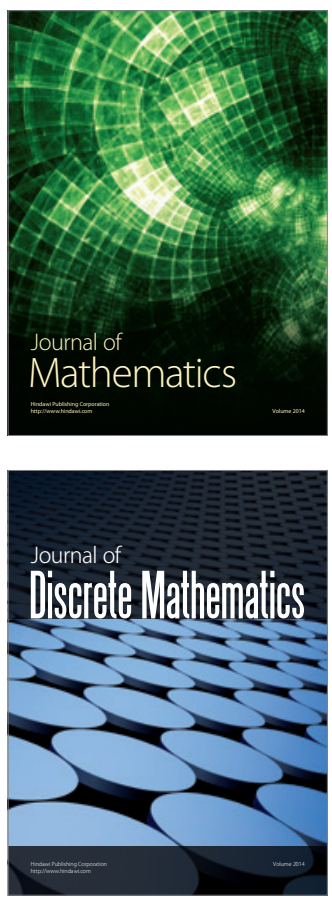

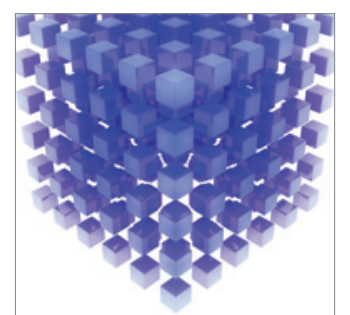

Mathematical Problems in Engineering
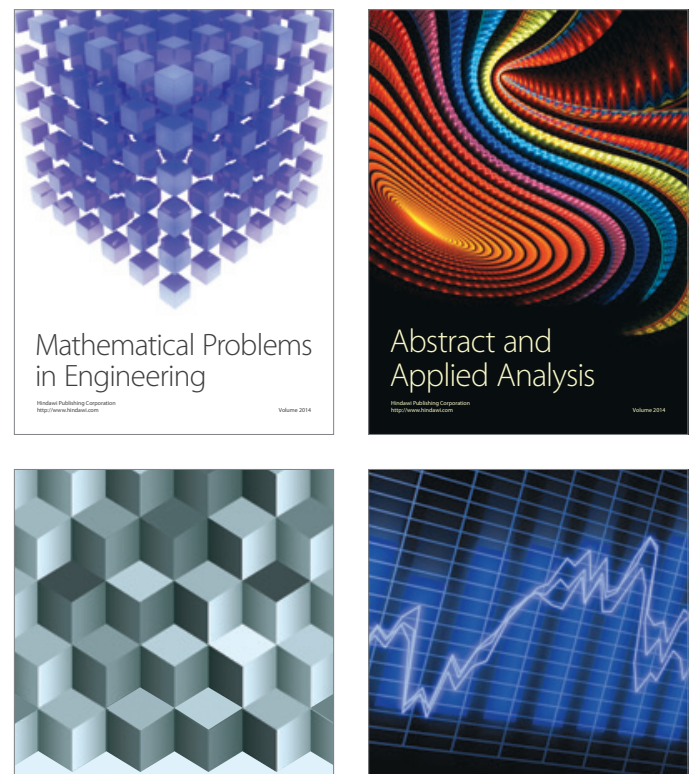

Journal of

Function Spaces

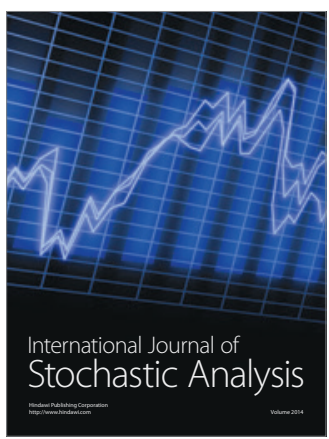

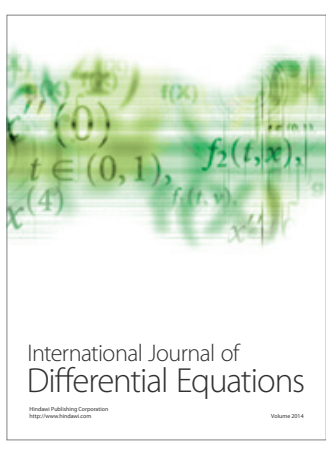
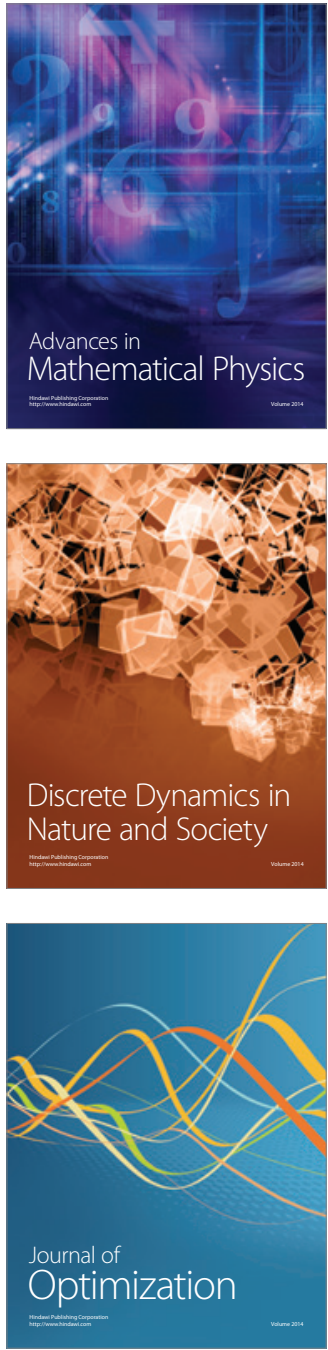